\section{Gliomas y neurofibromatosis tipo 1 Gliomas and neurofibromatosis type 1}

Como muy bien señalan el Dr. Aguirre y col, en su Comunicación Corta (1) a propósito de un glioma de nervio óptico en un niño con neurofibromatosis infantil tipo 1 , tanto el protocolo diagnóstico como terapéutico en gliomas de nervio y vías ópticas es controvertido.

Como la experiencia en esta patología, excepto en unidades altamente especializadas, a menudo es anecdótica; conviene tener protocolos o guías de actuación a las que acogerse para compensar la escasa experiencia individual. En este sentido, parece oportuno reseñar algunos de los comentarios de una revisión, publicada con posterioridad a esta comunicación, y que pueden sernos de gran ayuda para enfrentarnos a esta patología (2).

Respecto al protocolo diagnostico oftalmológico en el contexto de una neurofibromatosis, el "NF1 Optic Pathway Glioma Task Forced" recomienda realizar una exploración oftalmológica completa (incluyendo agudeza visual, examen biomicroscópico, fondo de ojo, visión cromática y campo visual) anualmente en menores de 6 años. Dada la escasa tasa de progresión después de esta edad, a partir de los 6 años las revisiones oftalmológicas se espaciarían (como protocolo orientativo pueden realizarse a los 8,10,16,20 y 25 años), salvo que aparecieran alteraciones. Respecto al tratamiento no existe un protocolo consensuado. Teniendo en cuenta la posibilidad de regresión espontánea en algunos casos y el riesgo de tumores secundarios asociados al tratamiento, el tratamiento agresivo en casos asintomáticos es más que controvertido. Sí estaría indicado en cambio ante una perdida visual asociada severa $(<0,5)$, o cuando existen signos de progresión como hipertensión intracraneal o déficit motor asociado (3).

La opción terapéutica varía en función de la edad; de modo que en menores de 5 años es electiva la poliquimioterapia, mientras que la radioterapia se reserva para mayores de esta edad. La cirugía es una alternativa en tumores aislados de nervio óptico.

En cualquier caso, para poder ponderar adecuadamente el papel de la cirugía, radio y quimioterapia en esta patología, deberemos esperar a los resultados de un ensayo clínico desarrollado en este sentido por la Organización Europea para la Investigación y tratamiento del Cáncer.

Rebolleda G

Hospital Ramón y Cajal. Madrid E-mail: grebolleda@telefonica.net

\section{BIBLIOGRAFÍA}

1. Aguirre-Balsalobre FE, Coloma-Gonzalez IT, MengualVerdu EN. Glioma de nervio óptico en un caso de neurofibromatosis-1 infantil. Arch Soc Esp Oftalmol 2006; 81: 33-36.

2. Sylvester CL, Drohan LA, Sergott RC. Optic nerve gliomas, chiasmal gliomas and neurofibromatosis type 1. Curr Opin Ophthalmol 2006; 17: 7-11

3. Listernick R, Louis DN, Packer RJ, Gutmann DH. Optic pathway gliomas in children with neurofibromatosis 1: consensus statement from the NF1 Optic Pathway Glioma Task Force. Ann Neurol 1997; 41: 143-149. 